\title{
Etched and nanocoated single-mode multimode single-mode (SMS) fibers for detection of wind turbine gearbox oil degradation
}

\author{
Ignacio Del Villar, Jaime Goñi, Adrian Vicente, Francisco J. Arregui and Ignacio R. Matias
}

\begin{abstract}
The application of an etching process in a singlemode multimode single-mode (SMS) fiber allows monitoring the change of refractive index of wind turbine gearbox oil due to temperature and degradation with a limitation at short temperatures, where the transmission and attenuation bands in the optical spectrum fade. The application of a black tin oxide nanocoating solves this issue and allows tuning the refractive index region where the sensitivity is maximum. The SMS was designed for operating at short wavelengths, where the setup is less expensive. The experimental results were contrasted with a theoretical analysis developed with FIMMWAVE, which allowed understanding better the phenomena involved in the experiments.

Index Terms - optical fiber sensors, single-mode multimode single-mode (SMS), low cutoff single-mode fiber, thin films, wind turbine prognosis.
\end{abstract}

\section{INTRODUCTION}

$\mathrm{T}$ he application of nanodepositions in the domain of optical fiber sensors has allowed this technology to be positioned in the domain of gas, chemical and biological sensors [1], [2], [11]-[13], [3]-[10]. The explanation is simple. The optical fiber transmits light, which can be modulated by parameters that affect its guidance through the optical fiber, such as strain, temperature or surrounding medium refractive index [14], [15]. However, if the deposition of a material on the optical fiber modulates the transmission of light through the fiber, these sensitive materials will modulate the transmission of light through the optical fiber as a function of almost any parameter (i.e. any environmental variables, chemical or biological species, etc.). Moreover, nanotechnology is evolving so much that a lab on fiber can be developed with optimized sensitivity to one or several parameters [16].

Another interesting property of nanodeposition is that new optical phenomena can be obtained by appropriately selecting the parameters of the coating (i.e. refractive index and thickness). One good example is the mode transition in longperiod fiber gratings, which causes an enhancement of the refractive index sensitivity [17]-[19]. In other structures, such as nanocoated tilted fiber Bragg gratings [20] or nanocoated nanoring resonators [21], this phenomenon is not explicitly

This work was supported in part by the Spanish Ministry of Education and Science-FEDER TEC 2016-78047 and by the Government of Navarre through its project with reference: 2017/PI044.

I. Del Villar, J. Goñi, A. Vicente, F. J. Arregui and I. R. Matias are with the Electrical and Electronic Engineering Department of the Public University of mentioned but also a sensitivity increase is obtained. Moreover, the mode transition has also been observed in single-mode multimode single-mode (SMS) fiber [22].

In this work, focus will be centered precisely on the latter structure, the SMS fiber, which is one of the simplest and most cost-effective optical fiber platforms that can be used in the domain of sensors since it basically consists of splicing two pigtails of single-mode fiber (SMF) to a coreless optical fiber. Contrary to other works, where standard SMF is used, here SMF operating at short wavelengths is selected, which allows monitoring the wavelength range from 400 to $1000 \mathrm{~nm}$, where optical sources and detectors are less expensive. In addition, in order to improve and to tune the device sensitivity, etching and thin film deposition will be applied to the structure, which will serve for using the device in a demanding application: lubricant oil testing, which allows judging a machine's health condition and providing a prognosis of the machine's failure progression. In the domain of wind farms, this is essential for reducing maintenance costs, and, hence, it has attracted much interest during the last years [23]. Different parameters can be used for testing the quality of lubricant oil for the sake of detecting air content, water content or even particle content in the oil [24], [25]. These parameters are basically the density, the viscosity and the refractive index of the liquid [24]. The latter parameter, refractive index, has been recently explored by tracking the resonance bands obtained in the transmission spectrum of the SMS structure, with the aim of studying the oil degradation or the influence of temperature on this parameter [26]. Here, a more thorough analysis is offered, which includes the effect of thin film nanodeposition along with a theoretical analysis based on FIMMWAVE. This allows tuning the sensitivity and controlling the fading of transmission and attenuation bands. In Section 2, the experimental setup and the methods used for simulating, fabricating and monitoring the SMS structure are described. In Section 3, the experimental results are detailed. Finally, some conclusions are presented in Section 4.

\section{METHODS AND MATERIALS}

The four types of structures analyzed in this work are

Navarra and the Institute of Smart Cities, 31006 Pamplona, Spain (e-mail: ignacio.delvillar@unavarra.es).

Copyright (C) 2019 IEEE 
described in Fig. 1: a standard SMS fiber, an etched SMS structure, a thin-film coated SMS structure and an etched and thin-film coated SMS structure. All of them consist basically of a coreless multimode fiber (MMF) segment that is spliced to two single-mode fibers. In the case of the etched SMS structure, it is immersed in hydrofluoric acid (HF), and the diameter of the structure is progressively reduced as a function of the etching time. This diameter reduction aims at improving the device sensitivity [27]. Another strategy for increasing the sensitivity is the deposition of a thin film on the coreless region [28]. Moreover, both etching and thin-film deposition can be combined for a highly sensitive SMS structure [29]. (a) SMS structure

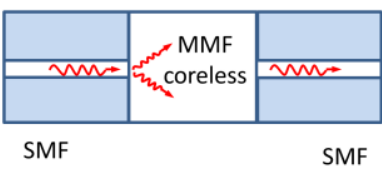

(b) Etched SMS structure

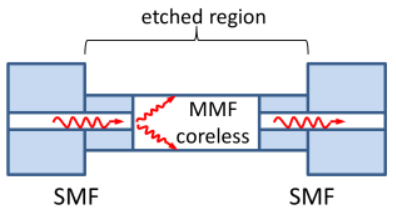

(c) Thin-film coated SMS structure

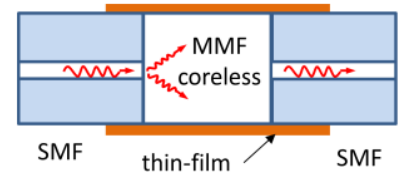

(d) Etched and thin-film coated SMS structure

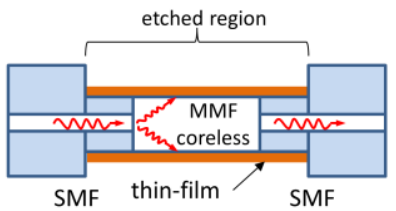

Fig. 1. (a) Standard SMS structure consisting of a coreless segment spliced to two SMF segments, (b) Etched SMS structure, (c) Thin-film coated SMS structure, (d) Etched and thin-film coated SMS structure.

The influence of etching will be analyzed by comparing two structures: one without etching and another with a 45-minute immersion in a $40 \%$ HF solution. In Fig. 2, the diameter of both structures is compared, and a diameter reduction from $125 \mu \mathrm{m}$ down to $31 \mu \mathrm{m}$ is observed.

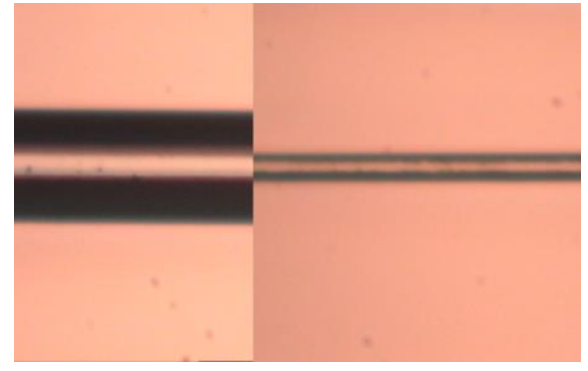

Fig. 2. Microscope image for a $125 \mu \mathrm{m}$ fiber before and after etching. The diameter was reduced down to $31 \mu \mathrm{m}$.

Regarding the thin-film coated structures, the material selected for the deposition was black tin oxide $\left(\mathrm{SnO}_{2-\mathrm{x}}\right)$, a material that is conductive and can be deposited with DC sputtering.

The $\mathrm{SnO}_{2-\mathrm{x}}$ target (57 $\mathrm{mm}$ in diameter and $3 \mathrm{~mm}$ in thickness) was purchased from ZhongNuo Advanced Material Technology Co. This material presents a higher refractive index than silica does and has been used for highly sensitive devices [30]. $\mathrm{SnO}_{2-\mathrm{x}}$ was deposited on three SMS structures with a DC sputtering device (K675XD from Quorum Technologies, Ltd.) at $90 \mathrm{~mA}$ current and $8 \times 10^{-2}$ mbar pressure. The deposition time used for each SMS structure was 25,35 and 45 seconds respectively.

The thickness of the coatings was analyzed with a field emission scanning electron microscope (FESEM) UltraPlus, from Carl Zeiss Inc. with an in-lens detector at $3 \mathrm{kV}$ and an aperture diameter of $30 \mu \mathrm{m}$. In Fig. 3, it can be observed that the thickness increases as a function of the deposition time: the thickness values obtained for 25,35 and 45 seconds of deposition were respectively 55,67 and $89 \mathrm{~nm}$.

\section{(a)}

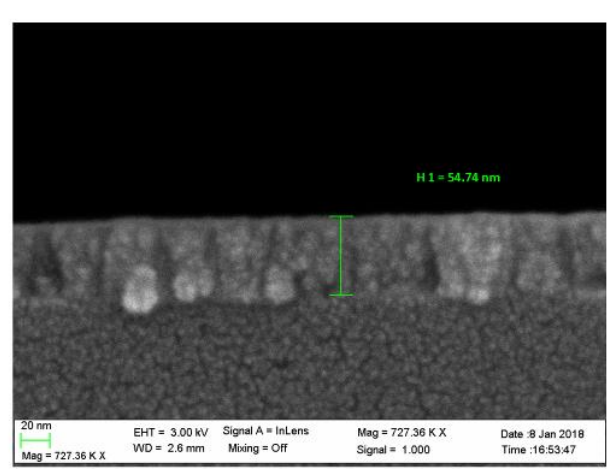

(b)

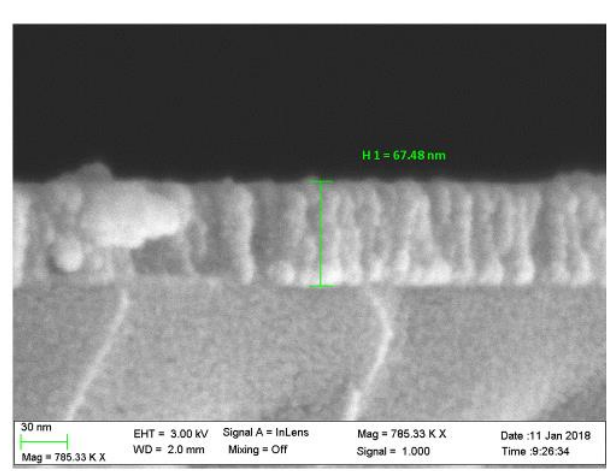

(c)

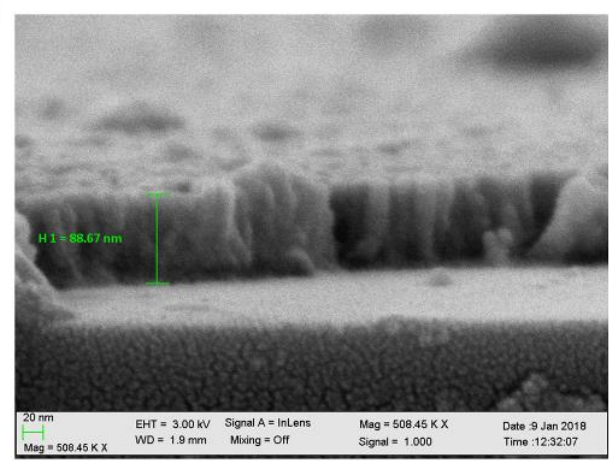

Fig. 3. FESEM image of the cross-section of an SMS structure coated for (a) 25 seconds, (b) 35 seconds, (c) 45 seconds.

For the single-mode section, a low cutoff wavelength fiber was selected (S630 fiber from Thorlabs with core diameter 3.5 $\mu \mathrm{m}$, cladding diameter $125 \mu \mathrm{m}$ and $\mathrm{NA}=0.12$ ), which allowed operating at short wavelengths $(630-860 \mathrm{~nm})$, where less expensive optical sources and detectors can be used. Regarding the multimode section, it consisted of a $20 \mathrm{~mm}$ long segment of an NCF125 coreless fiber from POFC Inc. (Taiwan), where 
light transmitted directly interacts with the outer medium, in this case the lubricant oil. The material of the coreless fiber was silica.

All SMS structures were connected on one end to an ASBNW tungsten-halogen broadband source from Spectral Products Inc. The output of the SMS structure was connected to an HR4000 spectrometer from OceanOptics, which was used for monitoring the transmission spectrum during the etching process and while the SMS structure was immersed in Fuchs Renolin CLP 320 lubricant oil (see Fig. 4). Two samples of this lubricant oil were used: one fresh and the other one degraded after 70,000 hours of utilization in the gearbox of a wind turbine. The refractive indices of these two samples were measured with a refractometer Mettler Toledo Refracto 30GS at a wavelength of $589 \mathrm{~nm}$, and the values obtained were respectively 1.467 and 1.4678 .

For the oil characterization, the following procedure was followed. The solution was heated up to $110{ }^{\circ} \mathrm{C}$. Then, the heating process was stopped, and multiple spectra were collected while the oil solution was spontaneously cooled. At the same time that the optical spectra were collected, a PT100 sensor connected to a 34970A Data Acquisition/Data Logger Switch Unit was used to register the temperature corresponding to each optical spectrum.

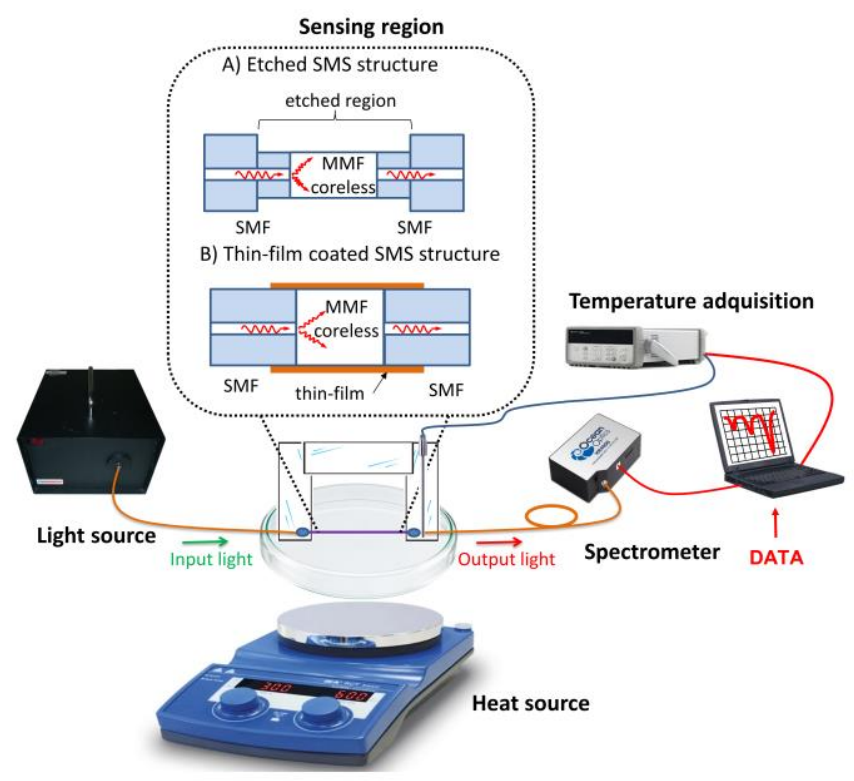

Fig. 4. Experimental setup: etched and thin-film SMS structures were used for testing the effects of temperature and aging of Fuchs Renolin CLP 320 lubricant oil.

The theoretical analysis was performed with FIMMAVE®. The propagation was obtained with FIMMPROP, a module integrated with FIMMWAVE. The finite difference method FDM was used for the SMF and MMF sections, since it is the most accurate method available for cylindrical waveguides. In the SMF sections only, the fundamental mode was analyzed, whereas for the MMF section 50 modes were analyzed, thus allowing to achieve convergence in the results. For surrounding medium refractive indices above the refractive index of the coreless section, a perfectly matched layer (PML) was used.

\section{RESULTS}

\section{A. Sensitivity increase with diameter reduction}

The refractive index sensitivity in SMS fibers is inversely proportional to the diameter of the coreless section [27]. Consequently, a sensitivity improvement factor of 4 should be obtained if we compare the performance of the SMS structures of $125 \mu \mathrm{m}$ (SMS without etching) and $31 \mu \mathrm{m}$ (SMS with 45minute etching) described in Section 2. This prediction is confirmed with the results presented in Figs. 5 and 6.

In Fig. 5, the optical spectrum of both unetched and etched structures is presented for three different surrounding refractive indices: $1.3333,1.3657$ and 1.4029. It is evident that a higher wavelength shift is achieved with the SMS fiber of lower diameter.
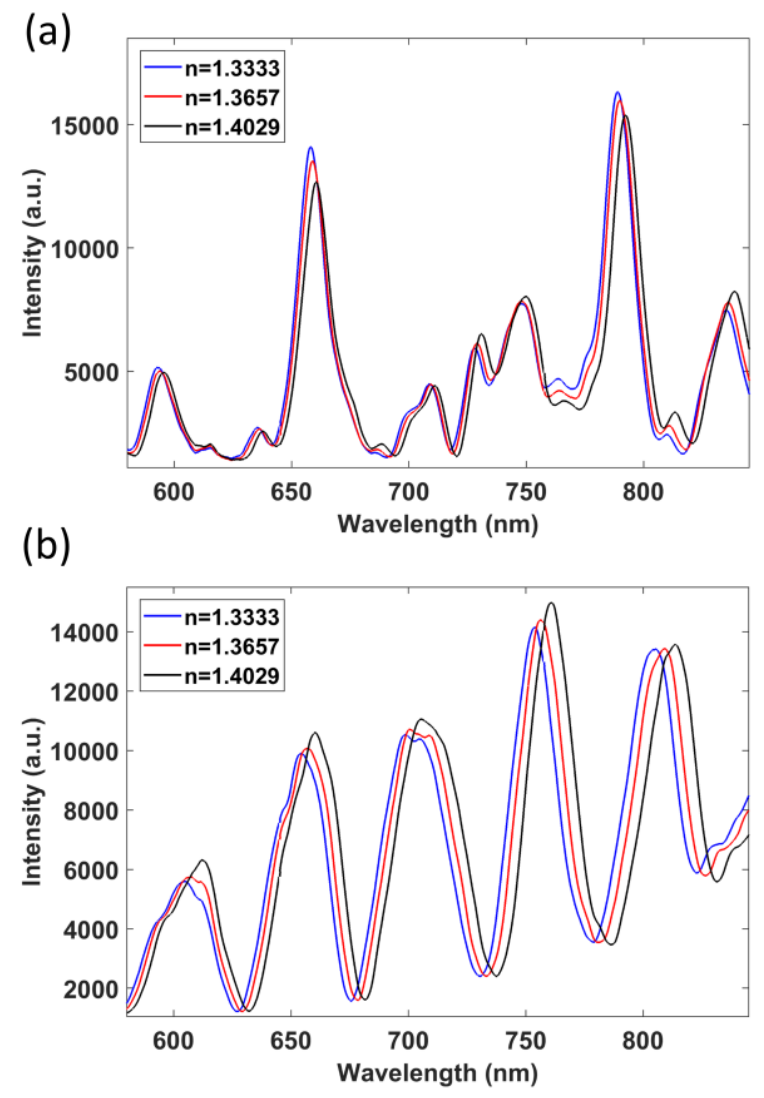

Fig. 5. Transmission optical spectra with different surrounding refractive indices [n]: (a) SMS without etching (fiber diameter $125 \mu \mathrm{m}$ ); (b) SMS with etching (fiber diameter $31 \mu \mathrm{m}$ ).

In addition to this, in Fig. 6 one maximum in the optical spectra of Fig. 5 is monitored as a function of refractive index for both structures. For the case of the SMS structure with diameter $125 \mu \mathrm{m}$, the maximum at $789 \mathrm{~nm}$ was tracked, whereas for the SMS structure with diameter $31 \mu \mathrm{m}$ the maximum at $753 \mathrm{~nm}$ was monitored. The purpose of selecting similar wavelengths was to avoid the influence of the wavelength on the sensitivity. The results in Fig. 6 show that the relative wavelength shift for the SMS structure with diameter $31 \mu \mathrm{m}$ is approximately 4 times the wavelength shift of the SMS structure with diameter $125 \mu \mathrm{m}$. This idea is also 
confirmed with the theoretical results presented in Fig. 7, where FIMMWAVE software was used with the parameters indicated in Section 2.

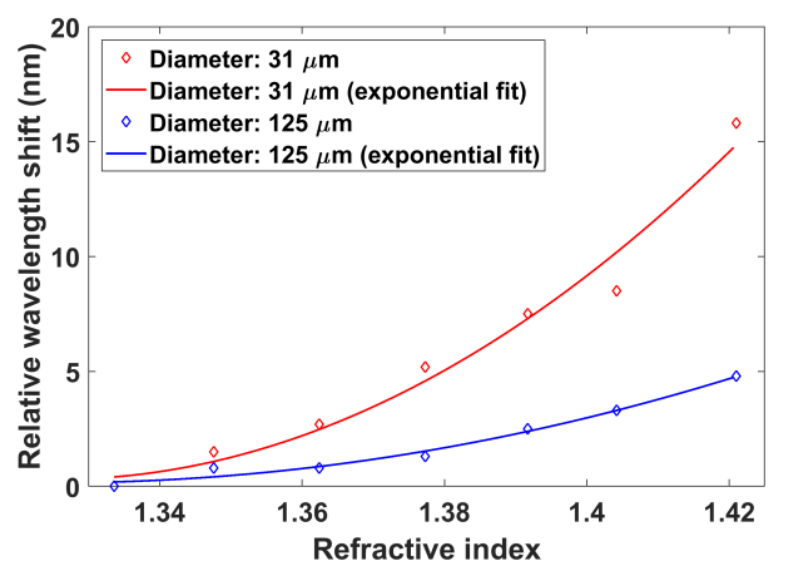

Fig. 6. Experimental results: relative wavelength shift with refractive index for SMS without etching (fiber diameter $125 \mu \mathrm{m}$ ) and for SMS with etching (fiber diameter $31 \mu \mathrm{m})$.

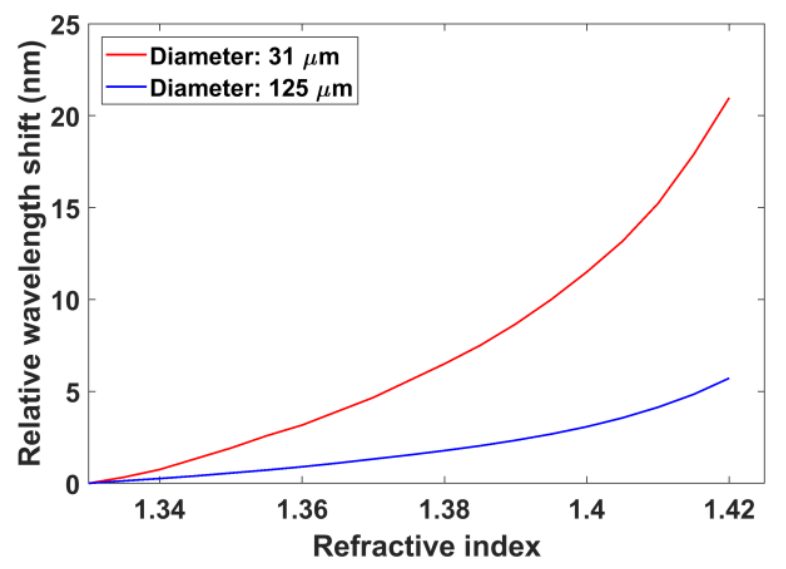

Fig. 7. Theoretical results: relative wavelength shift with refractive index for SMS without etching (fiber diameter $125 \mu \mathrm{m}$ ) and for SMS with etching (fiber diameter $31 \mu \mathrm{m})$.

After that, both sensors were immersed in fresh lubricant oil, and the optical spectrum was monitored with the method explained in Section 2, which permitted to obtain the curves presented in Fig. 8.

The results in Fig. 8 (transmitted optical power as a function of temperature and wavelength) show that the different bands in the optical spectrum experience a wavelength shift to the blue as temperature increases. According to Figs. 6 and 7, this indicates that the refractive index decreases at higher temperatures. Moreover, if we compare the results obtained with or without etching, it is evident that a higher wavelength shift is observed with etching, according to the higher sensitivity to refractive index observed in Figs. 6 and 7.

In addition, the SMS structure that shows a higher sensitivity was immersed in degraded oil (see Fig. 9), and a similar wavelength shift is observed. However, the region of highest sensitivity when the sensor is immersed in fresh oil is located at
$55-60{ }^{\circ} \mathrm{C}$, whereas for degraded oil this region is located at 58$63{ }^{\circ} \mathrm{C}$. This indicates that the refractive index is higher for degraded oil, according to [31].

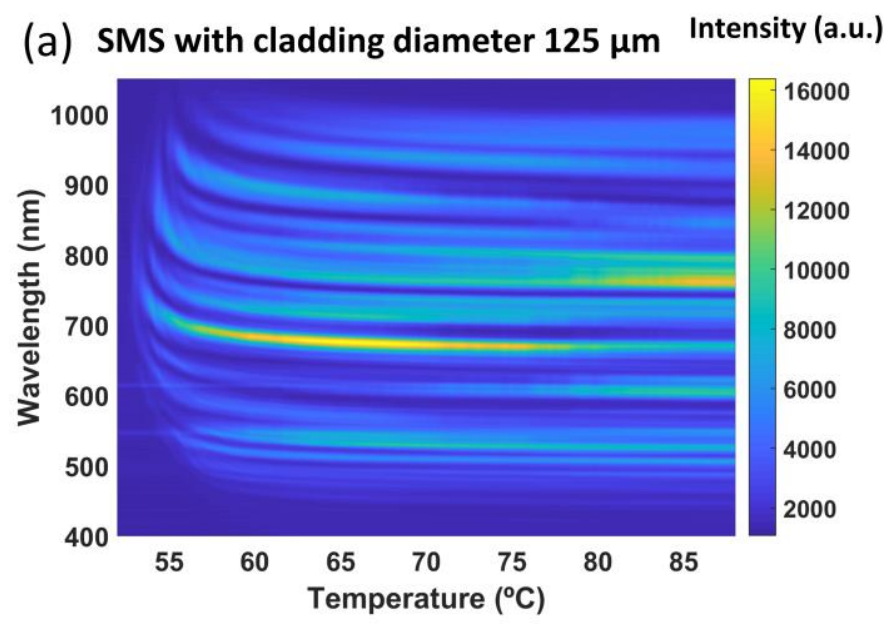

(b) SMS with cladding diameter $\mathbf{3 1} \boldsymbol{\mu m} \quad$ Intensity (a.u.)

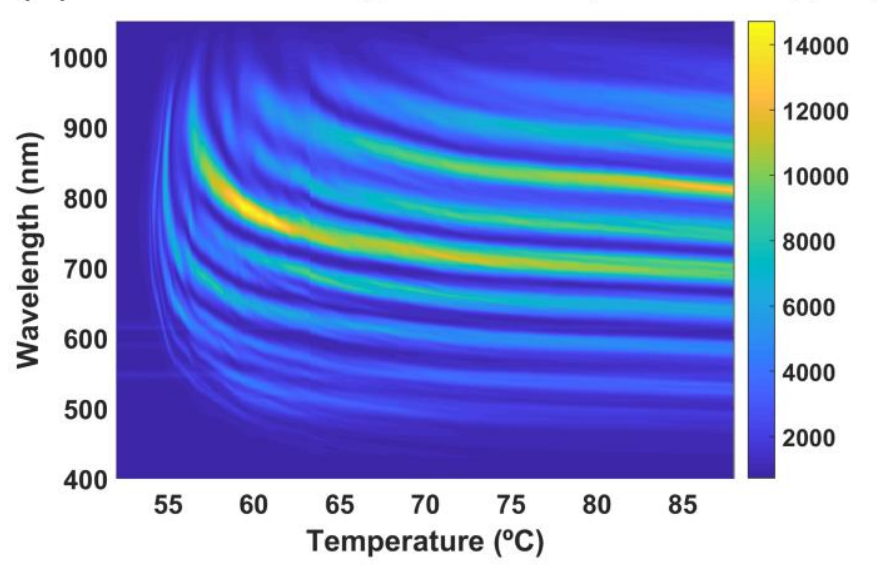

Fig. 8. Evolution of the optical spectrum as a function of temperature of an SMS structure immersed in fresh lubricant oil: a) without etching (diameter $125 \mu \mathrm{m}$ ); b) without etching (diameter $125 \mu \mathrm{m}$ ).

On the other hand, at low temperatures the refractive index of the solution approaches that of silica, and the transmission bands disappear after the highest sensitivity is attained, something that is observed in Figs. 8 and 9. This indicates that the refractive index of the oil is higher than that of the optical fiber structure. Consequently, the modes in the coreless section become radiation modes and no power is transmitted, which prevents the utilization of the sensor for temperatures below 55 ${ }^{\circ} \mathrm{C}$.

In Fig. 10, the wavelength of four maxima in the spectra of Fig. $8 \mathrm{~b}$ and Fig. 9 are tracked (i.e. the same SMS structure immersed in fresh and degraded lubricant oil). This proves the ability of this sensor to be used as a wavelength-based sensor for detecting both degradation and oil temperature, with the above-mentioned limitation of operation at temperatures below $55^{\circ} \mathrm{C}$. 


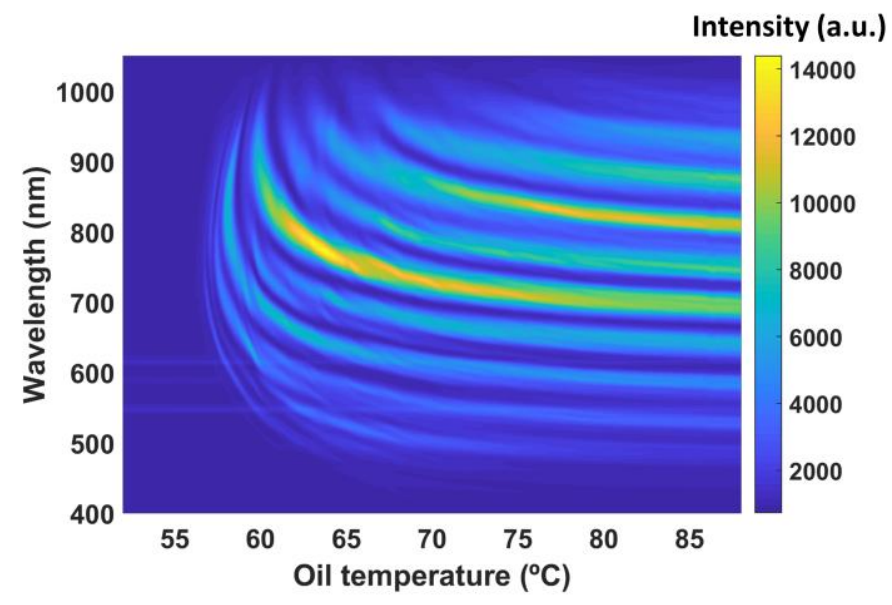

Fig. 9. Evolution of the optical spectrum as a function of temperature when the SMS structure with etching (diameter $31 \mu \mathrm{m}$ ) is immersed in degraded lubricant oil after 70,000 hours of utilization.

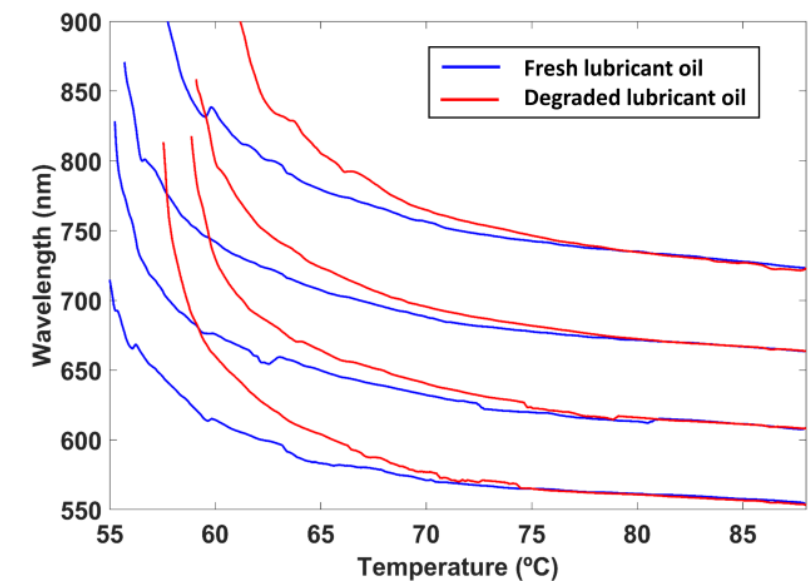

Fig. 10. Wavelength shift of some transmission bands of Figs. $8 \mathrm{~b}$ and 9 , corresponding to the same sensor immersed in fresh and in degraded lubricant oil.

\section{B. Tuning the working point of the sensor with a nanocoating}

In order to expand the applicability of this sensor, it is necessary to increase the range of operation of the sensor by tuning its working point. To this purpose, nanocoatings of different thickness will be deposited on the cladding of the SMS structure. For the sake of simplicity, focus will be centered only on SMS structures without etching, and the results will be compared with those obtained with the same SMS structure but without the thin film.

In Fig. 11, the SMS structure without thin film is analyzed for a wide range of refractive indices. The relative wavelength shift of a minimum located at $720 \mathrm{~nm}$ is tracked for refractive indices ranging from 1.33 to 1.65 . The results are compared with those obtained with FIMMWAVE, according to the method presented in Section 2, and a global agreement was found between the experimental and theoretical data. The mismatch between the experiment and the simulation is probably due to not considering the dispersion of the refractive index solutions used during the experiments.

It must be highlighted that the region of highest sensitivity is located in the vicinity of refractive index 1.45 , which is the index of the silica coreless segment. This sensitivity increase agrees well with the behavior observed in Fig. 8 when the temperature is approximately $55^{\circ} \mathrm{C}$, and at the same time there is a decrease of the signal intensity, which prevents using the sensor for temperatures below $55^{\circ} \mathrm{C}$. Indeed, this behavior was also observed when obtaining the experimental points of Fig. 11. It was not possible to obtain the point corresponding to refractive index 1.464 , a value very similar to the refractive index 1.467 obtained with the Mettler Toledo refractometer at $23{ }^{\circ} \mathrm{C}$. This indicates that the refractive index at temperature 55 ${ }^{\circ} \mathrm{C}$ must be approximately $1.46-1.47$.

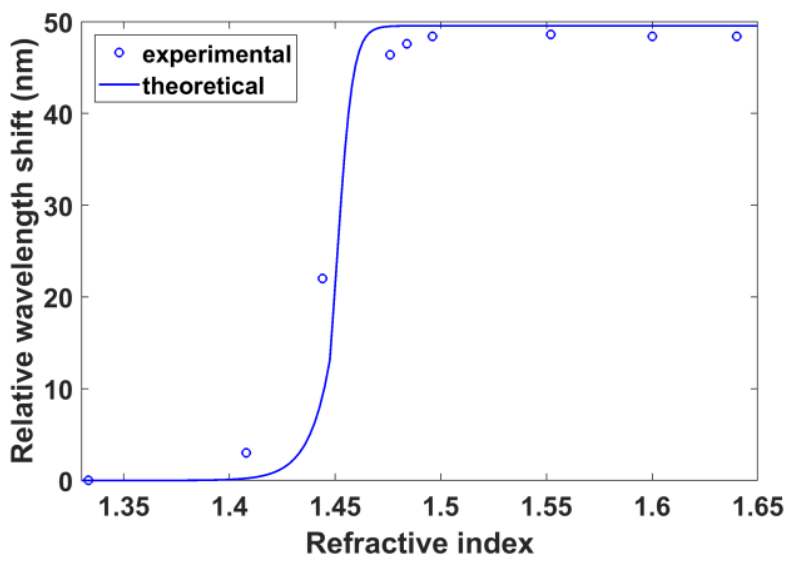

Fig. 11. Experimental (circles) and theoretical (solid line) relative wavelength shift with refractive index for SMS without etching (diameter $125 \mu \mathrm{m}$ ) and without nanocoating deposition.

If SMS structures without etching are deposited with thin films of 55, 67 and $89 \mathrm{~nm}$ (see Fig. 3), the region of highest sensitivity is shifted to a shorter refractive index and no fading of the spectrum is observed. In Fig. 12, the relative wavelength shift for each SMS structure is represented. It must be pointed out that the wavelength shift is now combined with the mode transition, which increases the device sensitivity at the same time that it makes tracking the relative wavelength shift as a function of the refractive index to be more difficult. Consequently, no points in the region of highest sensitivity are represented.

Finally, in Fig. 13 the refractive index with highest sensitivity for the three thin-film coated SMS structures and the uncoated SMS structure is represented, proving the idea that it is possible to tune the working point of the sensor by controlling the coating thickness.

The previous conclusion is also corroborated with theoretical results focused on the relative wavelength shift observed while decreasing the refractive index from 1.5 to smaller values (see Fig. 14). There, it is evident that the region of highest sensitivity is shifted to shorter refractive indices when a nanocoating is deposited, which will have some consequences in the way the sensor operates. 
(a) SMS sputtered with $\mathrm{SnO}_{2}$ for 25 seconds

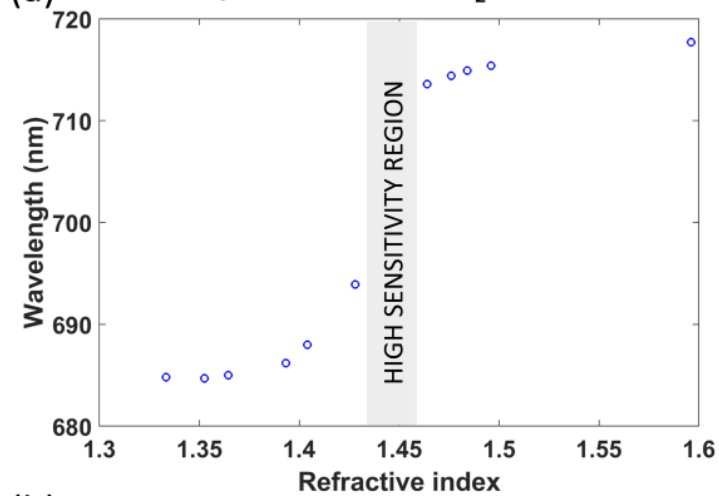

(b) SMS sputtered with $\mathrm{SnO}_{2}$ for 35 seconds

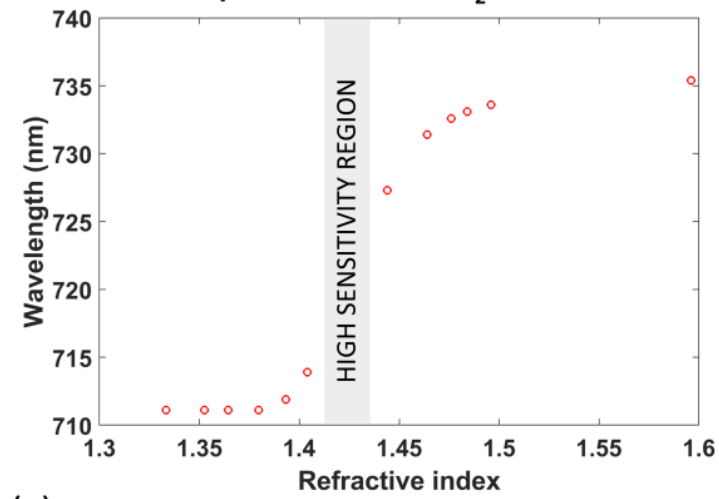

(c) SMS sputtered with $\mathrm{SnO}_{2}$ for 45 seconds

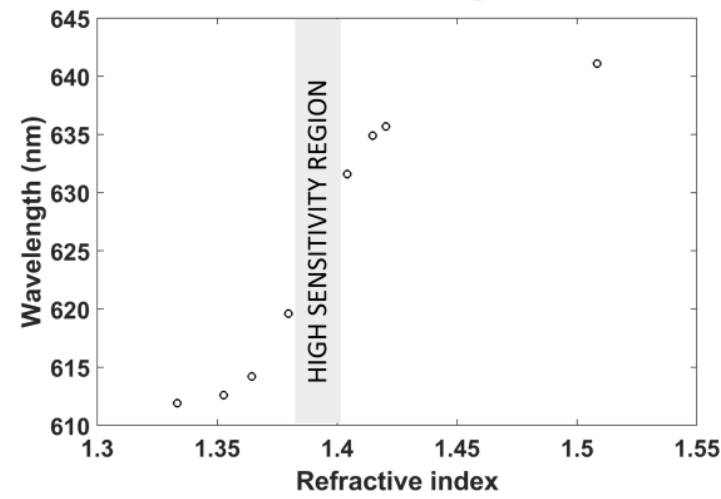

Fig. 12. Relative wavelength shift with refractive index for SMS with different $\mathrm{SnO}_{2-\mathrm{x}}$ deposition times: a) 25 seconds (thickness $55 \mathrm{~nm}$ ), b) 35 seconds (thickness $67 \mathrm{~nm}$ ), c) 45 seconds (thickness $89 \mathrm{~nm}$ ). The region of highest sensitivity is progressively shifted.

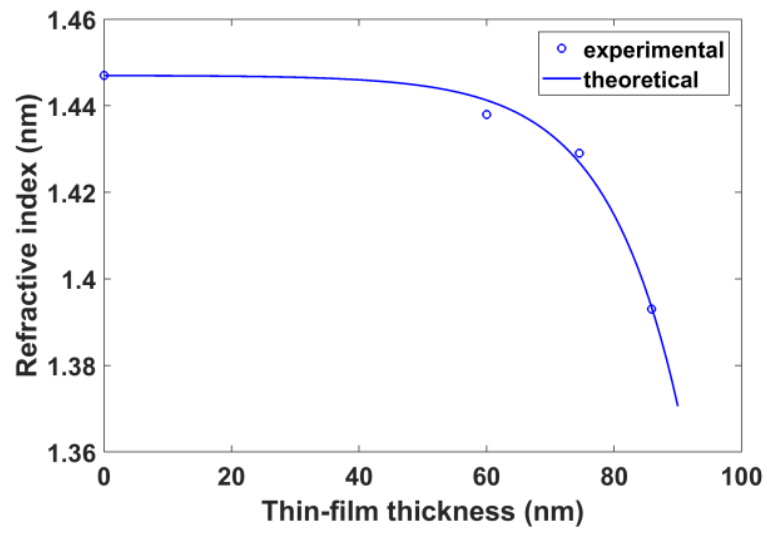

Fig. 13. Refractive index region of highest sensitivity as a function of the nanocoating thickness in an SMS structure of diameter $125 \mu \mathrm{m}$.

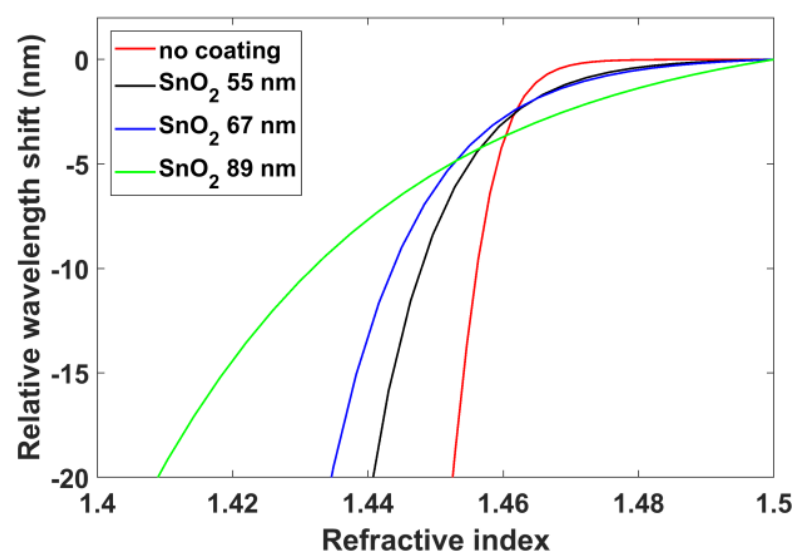

Fig. 14. Theoretical results for relative wavelength shift with refractive index for SMS with different nanocoating thicknesses: 0, 55, 67 and $89 \mathrm{~nm}$.

After the analysis of sensitivity to refractive index, the sensors are tested in lubricant oil. Fig. 15 shows the spectra for the two SMS structures analyzed in Fig 12: the one sputtered with $\mathrm{SnO}_{2-\mathrm{x}}$ for 25 seconds (coating thickness $55 \mathrm{~nm}$ ) and the one sputtered with $\mathrm{SnO}_{2-\mathrm{x}}$ for 35 seconds (coating thickness 67 $\mathrm{nm})$. A wider temperature range was monitored $\left(35-110^{\circ} \mathrm{C}\right)$ in order to prove the ability of the sensor to solve the limitation of uncoated sensors.

\section{(a) SMS sputtered with $\mathrm{SnO}_{2-\mathrm{x}} 25$ seconds Intensity (a.u.)}

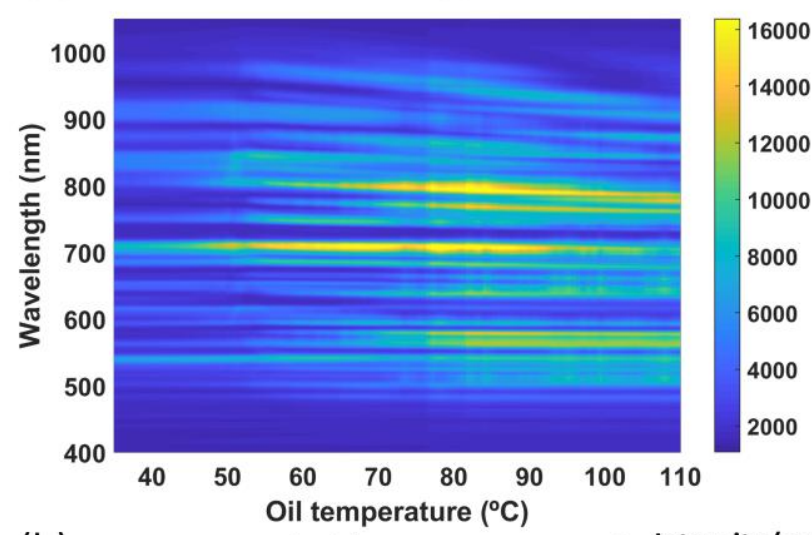

(b) SMS sputtered with $\mathrm{SnO}_{2-\mathrm{x}} 35$ seconds Intensity (a.u.)

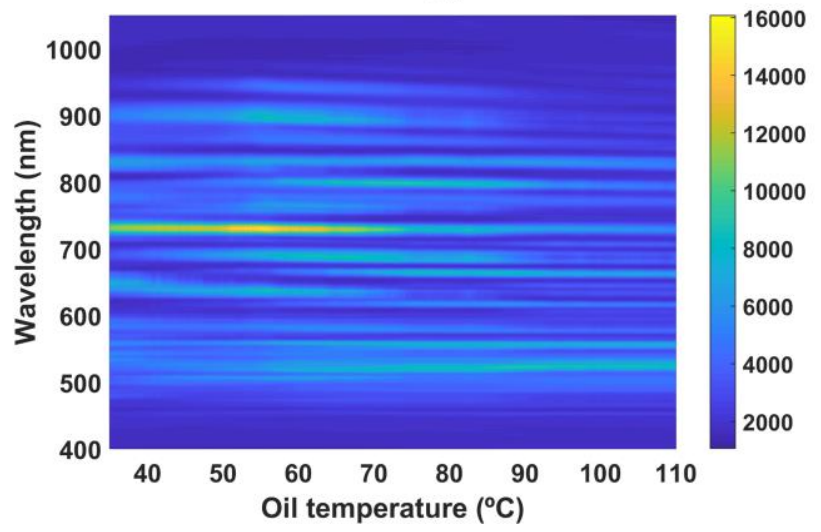

Fig. 15. Evolution of the optical spectrum as a function of temperature of an SMS structure without etching (diameter $125 \mu \mathrm{m}$ ) immersed in fresh lubricant oil and coated with different $\mathrm{SnO}_{2-\mathrm{x}}$ deposition times: a) 25 seconds (thickness $55 \mathrm{~nm}$ ), b) 35 seconds (thickness $67 \mathrm{~nm}$ ). 
So as to obtain a better comparison among the two sensors deposited with $\mathrm{SnO}_{2-\mathrm{x}}$ for 25 and 35 seconds and the uncoated sensor of Fig. 8a, in Fig. 16 the wavelength shift of one transmission band is analyzed for each structure. In order discard the influence of wavelength in the sensitivity, the closest band to $800 \mathrm{~nm}$ was selected in all cases.

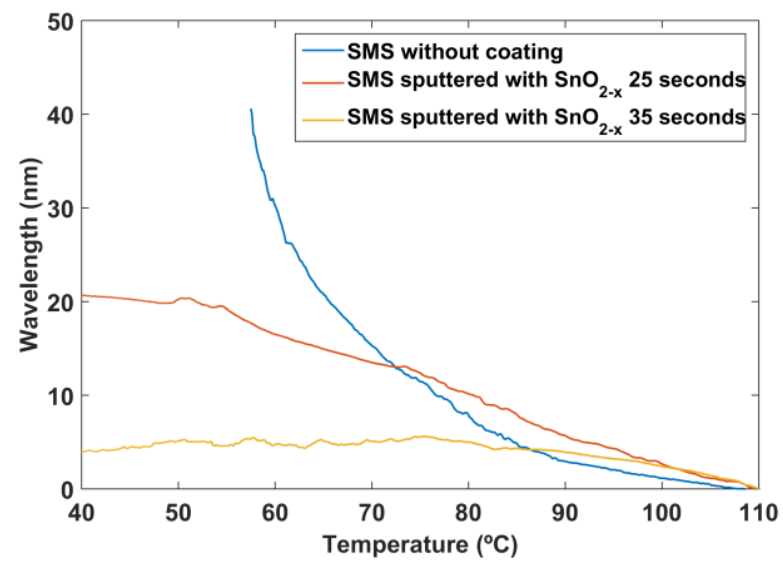

Fig. 16. Wavelength shift of evolution of the optical spectrum as a function of temperature of an SMS structure without etching (diameter $125 \mu \mathrm{m}$ ) immersed in fresh lubricant oil and coated with different $\mathrm{SnO}_{2-\mathrm{x}}$ deposition times: a) 25 seconds (thickness $55 \mathrm{~nm}$ ), b) 35 seconds (thickness $67 \mathrm{~nm}$ ).

The maximum sensitivity is lower than without coating (Fig. 8a) because, as stated above, the refractive index of Fuchs Renolin CLP 320 is approximately $1.45-1.47$, and for this region (see Fig. 14) the sensitivity of the coated sensors is lower compared to the case without coating. This is true because the high-sensitivity region is shifted to a lower refractive index for thin-film coated sensors. However, the good point is that now there is no fading of the resonances, which implies that the limitation in the temperature range of uncoated sensors has been overcome. This fading can be also understood by showing the spectrum obtained for the three sensors when immersed in refractive index 1.464. In Fig. 17, there are some transmission bands for the two nanocoated SMS structures whereas no bands are visible for the SMS structure without coating.

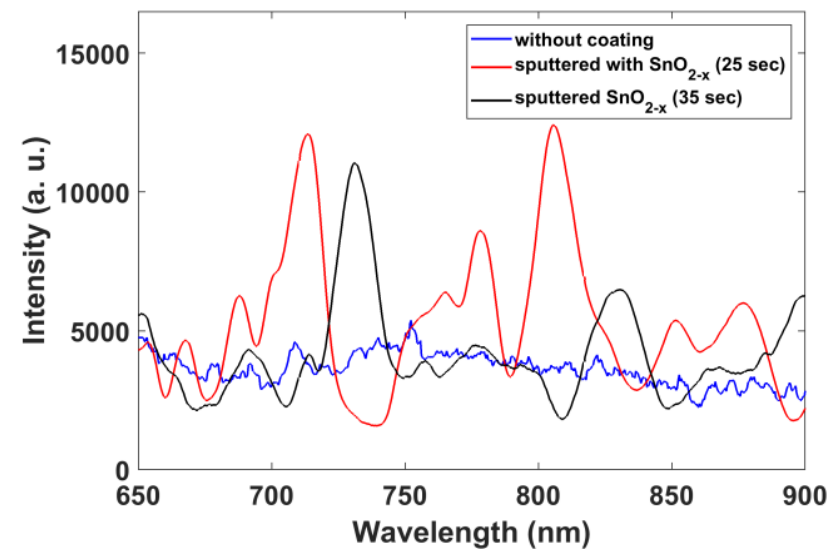

Fig. 17. Transmission optical spectra for three SMS structures without etching (diameter $125 \mu \mathrm{m}$ ) when immersed in refractive index 1.464. One of the structures presented no coating, and the other two were sputtered with $\mathrm{SnO}_{2-x}$ for 25 seconds (thickness $55 \mathrm{~nm}$ ) and 35 seconds (thickness $67 \mathrm{~nm}$ ).
In addition, if we compare in Fig. 16 both sensors deposited with $55 \mathrm{~nm}$ coating (sputtered for 25 seconds) and with $67 \mathrm{~nm}$ coating (sputtered for 35 seconds), there is a higher sensitivity when a thinner coating is deposited. In fact, it is difficult to use the SMS structure with thicker coating for sensing, whereas the $55 \mathrm{~nm}$ coated sensor presents a sensitivity of $0.3 \mathrm{~nm} /{ }^{\circ} \mathrm{C}$ in the range from 50 to $110{ }^{\circ} \mathrm{C}$ and $0.08 \mathrm{~nm} /{ }^{\circ} \mathrm{C}$ below $50{ }^{\circ} \mathrm{C}$. This better sensitivity is again due to the fact that the refractive index region of highest sensitivity is more separated in the case of a thicker coating.

In view of these results, a new experiment is presented where the combined effect of etching and deposition is analyzed (see Fig. 18). The purpose was to track the signal in a wide range of temperatures and at the same time to obtain a high sensitivity that permits to distinguish when the sensor is immersed in fresh and in degraded oil. This time, $\mathrm{SnO}_{2-\mathrm{x}}$ was sputtered for 20 seconds on an SMS structure etched during the same time that led to the diameter reduction presented in Fig. 2.

\section{(a) etched and nanocoated SMS immersed in fresh oil}

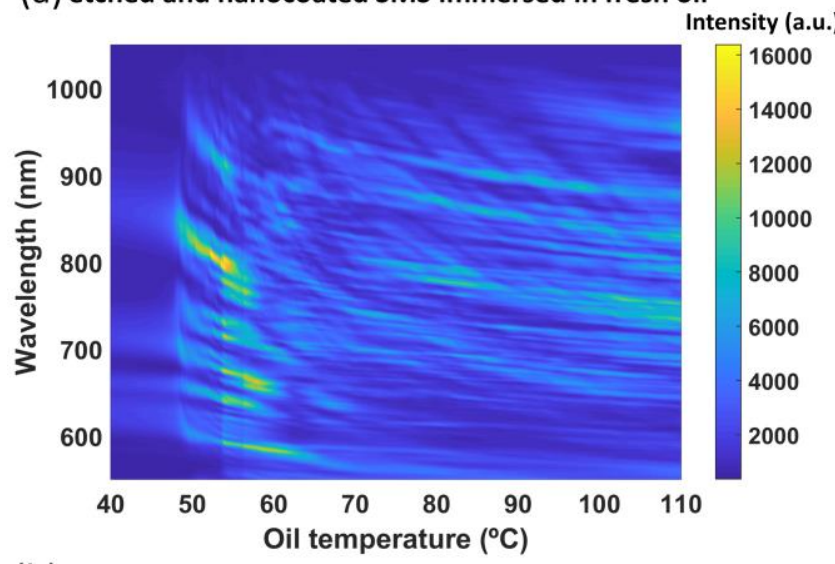

(b) etched and nanocoated SMS immersed in degraded oil

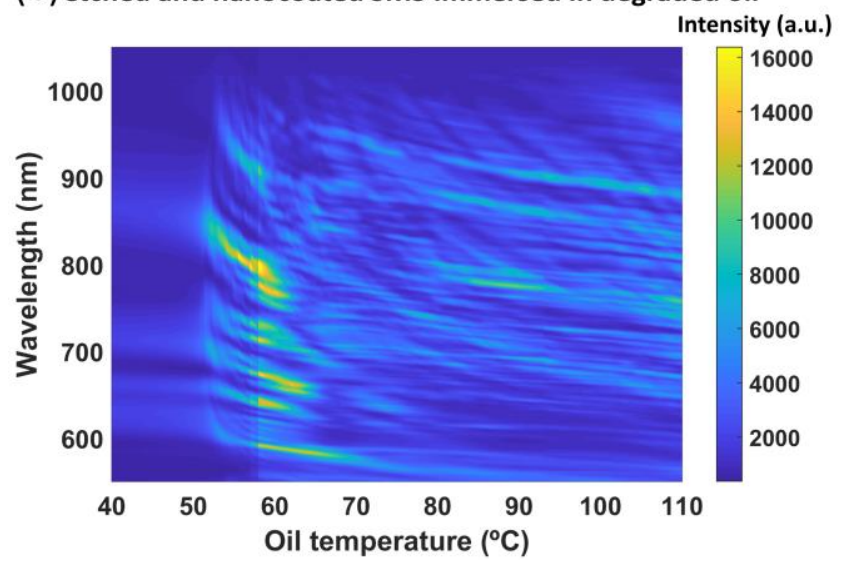

Fig. 18. Evolution of the optical spectrum as a function of temperature of an etched SMS structure sputtered with $\mathrm{SnO} 2-\mathrm{x}$ for 20 seconds: a) immersed in fresh lubricant oil; b) immersed in degraded lubricant oil.

It was stated above that this diameter reduction led to a sensitivity improvement of a factor of 4 , which is similar to the wavelength shift improvement that we see if we compare the blue line in Fig. 19 with the blue line in Fig. 16 ( $80 \mathrm{~nm}$ versus $20 \mathrm{~nm}$ overall wavelength). The sensitivity in Fig. 19 is 1.2 $\mathrm{nm} /{ }^{\circ} \mathrm{C}$ in the range from 50 to $110{ }^{\circ} \mathrm{C}$ and $0.4 \mathrm{~nm} /{ }^{\circ} \mathrm{C}$ below 50 ${ }^{\circ} \mathrm{C}$. The spectrometer used in the experiments presented a 
resolution of $0.25 \mathrm{~nm}$, which indicates that a resolution below $1{ }^{\circ} \mathrm{C}$ can be attained with the proposed sensor, which could be improved with a higher-resolution spectrometer. In addition, again it is possible to track transmission bands at temperatures below $50{ }^{\circ} \mathrm{C}$ (this was not possible with the uncoated sensor of Figs. 8-10). In Fig. 19, it is evident that below $50{ }^{\circ} \mathrm{C}$ there is a decrease of the wavelength shift rate, but enough to avoid the visibility of any band, as was the case in Fig. 8 and Fig. 9. It must be pointed out that the signal is worsened, probably due to the imperfect deposition of the sputtering process (atomic layer deposition would probably lead to better results). However, in spite of this it is possible to observe that the signals corresponding to immersion in fresh and degraded oil are actually shifted from each other, which indicates the detection of the change of refractive index in fresh and degraded oil.

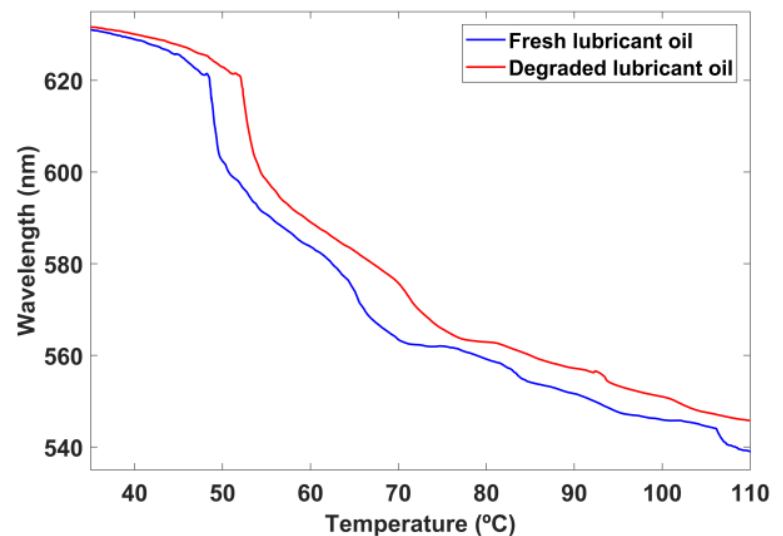

Fig. 19. Wavelength shift of a transmission band of Fig. 18, corresponding to the same etched and nanocoated sensor immersed in fresh and in degraded lubricant oil.

For the sake of comparison, a final etched sensor was developed with a thicker coating (sputtered for 35 seconds). According to what was observed in Fig. 16, a lower sensitivity was attained in Fig. $20\left(0.3 \mathrm{~nm} /{ }^{\circ} \mathrm{C}\right.$ in the range from 50 to 110 $\left.{ }^{\circ} \mathrm{C}\right)$. In addition, in order to test the repeatability of the sensor, it was analyzed two times in the range from 35 to $110^{\circ} \mathrm{C}$ (see the blue and the red plots in Fig. 20).

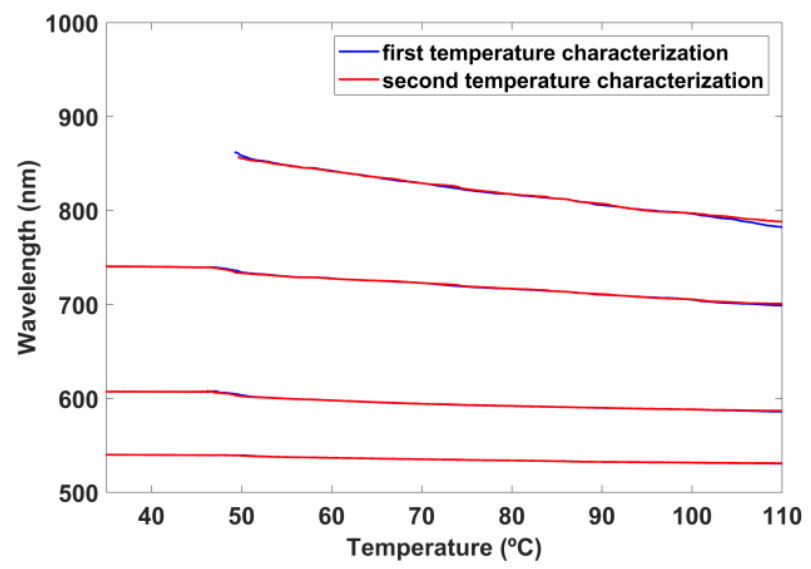

Fig. 20. Wavelength shift of some transmission bands in an etched and nanocoated sensor (sputtered for 35 seconds) as a function of temperature. The temperature analysis was repeated two times to test the performance of the sensor.

\section{CONCLUSION}

The utilization of an SMS fiber based on a coreless segment spliced to two low cutoff wavelength single-mode fibers permits operation at short wavelengths, where lights sources and detectors are less expensive.

This low-cost structure was used as a refractometer and for analyzing Fuchs Renolin CLP 320 wind turbine gearbox oil. In order to obtain the best performance, two strategies were followed. The first one was to reduce the structure diameter with an etching process, which allowed increasing the sensitivity of the device and being able to track more accurately the wavelength shift of the transmission and attenuation bands in the optical spectrum. However, when analyzing Fuchs Renolin CLP 320 wind turbine gearbox oil, a range of temperatures was found where the resonances fade, which prevents the utilization of the sensor in these conditions.

A second strategy based on the DC sputtering deposition of a $\mathrm{SnO}_{2-\mathrm{x}}$ thin film was used to prevent this fading problem. In this sense, an experimental and theoretical analysis of the wavelength shift of the bands as a function of refractive index allowed understanding that the thin-film thickness determines the refractive index region where a highest sensitivity is obtained. At the same time, the shift of the region of highest sensitivity prevents the effect of fading observed with the SMS without coating, which can be used for sensing in a wider range of temperatures at a cost of a reduction in sensitivity. As a final experiment, the effect of combining both the etching and deposition for a device of high sensitivity was tested, and it was proved that a wide range of operating temperatures can be tracked and even the difference between fresh and degraded oil can be detected. Consequently, it can be concluded that the ideas presented here prove that SMS structures can be tuned in terms of sensitivity and refractive index range where this high sensitivity is attained, which indicates the versatility of this simple and low-cost sensing structure.

\section{REFERENCES}

[1] F. Chiavaioli, F. Baldini, S. Tombelli, C. Trono, and A. Giannetti, "Biosensing with optical fiber gratings," Nanophotonics, vol. 6, no. 4, 2017.

C. Elosua, I. R. Matias, C. Bariain, and F. J. Arregui, "Volatile Organic Compound Optical Fiber Sensors: A Review," Sensors, vol. 6, no. 11, pp. 1440-1465, 2006.

[3] E. Benito-Peña, M. G. Valdés, B. Glahn-Martínez, and M. C. Moreno-Bondi, "Fluorescence based fiber optic and planar waveguide biosensors. A review," Anal. Chim. Acta, vol. 943, pp. 17-40, 2016.

[4] B. D. Gupta, S. K. Srivastava, and R. Verma, Fiber Optic Sensors Based on Plasmonics. World Scientific Publishing Co, 2015.

B. D. Gupta, A. M. Shrivastav, and S. P. Usha, "Surface plasmon resonance-based fiber optic sensors utilizing molecular imprinting," Sensors (Switzerland), 
vol. 16, no. 9, 2016.

[6] M. Pospíšilová, G. Kuncová, and J. Trögl, "Fiberoptic chemical sensors and fiber-optic bio-sensors," Sensors (Switzerland), vol. 15, no. 10, 2015.

[7] C. Caucheteur, T. Guo, and J. Albert, "Review of plasmonic fiber optic biochemical sensors: improving the limit of detection," Anal. Bioanal. Chem., vol. 407, no. 14 , pp. 3883-3897, 2015.

[8] A. Urrutia, J. Goicoechea, and F. J. Arregui, “Optical fiber sensors based on nanoparticle-embedded coatings," J. Sensors, vol. 2015, p. 805053, 2015.

[9] P. Vaiano et al., "Lab on Fiber Technology for biological sensing applications," Laser Photonics Rev., vol. 10, no. 6, pp. 922-961, 2016.

[10] D. Wencel, T. Abel, and C. McDonagh, "Optical chemical pH sensors," Anal. Chem., vol. 86, no. 1, pp. 15-29, 2014.

[11] X.-D. Wang and O. S. Wolfbeis, "Fiber-optic chemical sensors and biosensors (2008-2012)," Anal. Chem., vol. 85, no. 2, pp. 487-508, 2013.

[12] M. S. Luchansky and R. C. Bailey, "High-Q optical sensors for chemical and biological analysis," Anal. Chem., vol. 84, no. 2, pp. 793-821, 2012.

[13] J. Hodgkinson and R. P. Tatam, "Optical gas sensing: A review," Meas. Sci. Technol., vol. 24, p. 012004, 2013.

[14] B. Lee, "Review of the present status of optical fiber sensors," Opt. Fiber Technol., vol. 9, no. 2, pp. 57-79, 2003.

[15] S. W. James and R. P. Tatam, "Optical fibre longperiod grating sensors: characteristics and application," Meas. Sci. Technol., vol. 14, no. 5, pp. R49-R61, 2003.

[16] A. Ricciardi et al., "Lab-on-fiber technology: A new vision for chemical and biological sensing," Analyst, vol. 140, no. 24, pp. 8068-8079, 2015.

[17] I. Del Villar, I. R. Matias, S. Member, F. J. Arregui, and M. Achaerandio, "Nanodeposition of Materials With Complex Refractive Index in Long-Period Fiber Gratings," Lightwave, vol. 23, no. 12, pp. 4192-4199, 2005.

[18] I. Del Villar, M. Achaerandio, I. R. Matías, and F. J. Arregui, "Deposition of overlays by electrostatic selfassembly in long-period fiber gratings.," Opt. Lett., vol. 30, no. 7, pp. 720-722, 2005.

[19] A. Cusano et al., "Mode transition in high refractive index coated long period gratings.," Opt. Express, vol. 14, no. 1, pp. 19-34, 2006.

[20] J. Renoirt, C. Zhang, M. Debliquy, M. Olivier, and C. Caucheteur, "High-refractive-index transparent coatings enhance the optical fiber cladding modes refractometric sensitivity," Opt. Express, vol. 21, no. 23, pp. 29073-29082, 2013.

[21] A. B. Socorro, S. Soltani, I. Del Villar, J. M. Corres, and A. M. Armani, "Temperature sensor based on a hybrid ITO-silica resonant cavity," Opt. Express, vol. 23, no. 3, pp. 1930-1937, 2015.

[22] A. B. Socorro, I. Del Villar, J. M. Corres, F. J. Arregui, and I. R. Matias, "Mode transition in complex refractive index coated single-modemultimode-single-mode structure.," Opt. Express, vol. 21, no. 10, pp. 12668-82, 2013.

[23] X. Zhu, C. Zhong, and J. Zhe, "Lubricating oil conditioning sensors for online machine health monitoring - A review," Tribol. Int., vol. 109, no. January, pp. 473-484, 2017.

[24] D. A. Coronado and D. View, "Oil-sensors test bench : An approach to validate oil condition monitoring systems for wind turbine applications," Tribol. und Schmierungstechnik, vol. 63, no. 3, pp. 45-53, 2016.

[25] J. Zhu, J. M. Yoon, D. He, and Eric Bechhoefer, "Online particle-contaminated lubrication oil condition monitoring and remaining useful life prediction for wind turbines," Wind Energy, vol. 18, pp. 1131-1149, 2015

[26] I. Del Villar, J. Goñi, F. J. Arregui, and I. R. Matias, "Detection of wind turbine gearbox oil degradation with etched single-mode multimode single-mode (SMS) fiber," in Optical Fiber Sensors 2018, 2018, p. WF34.

[27] I. Del Villar, A. B. Socorro, J. M. Corres, F. J. Arregui, and I. R. Matias, "Refractometric sensors based on multimode interference in a thin-film coated singlemode-multimode-single-mode structure with reflection configuration," Appl. Opt., vol. 53, no. 18, pp. 3913-3919, 2014.

[28] A. B. Socorro, I. Del Villar, J. M. Corres, F. J. Arregui, and I. R. Matias, "Sensitivity enhancement in a multimode interference-based SMS fibre structure coated with a thin-film: Theoretical and experimental study," Sensors Actuators, B Chem., vol. 190, pp. 363-369, 2014.

[29] Y. Cardona, I. Del Villar, A. B. Socorro, J. M. Corres, I. R. Matias, and J. F. Botero-Calavid, "Wavelength and phase detection based SMS fiber sensors optimized with etching and nanodeposition," J. Light. Technol., vol. 35, no. 17, pp. 3743-3749, 2017.

[30] P. Sanchez, C. R. Zamarreño, M. Hernaez, I. R. Matias, and F. J. Arregui, "Optical fiber refractometers based on Lossy Mode Resonances by means of $\mathrm{SnO} 2$ sputtered coatings," Sensors Actuators, B Chem., vol. 202, pp. 154-159, 2014.

[31] P. Sánchez, C. R. Zamarreño, F. J. Arregui, and I. R. Matías, "LMR-based optical fiber refractometers for oil degradation sensing applications in synthetic lubricant oils," J. Light. Technol., vol. 34, no. 19, pp. 4537-4542, 2016. 